\title{
Chiral Symmetry Breaking Observed for Cysteine on the $\operatorname{Au}(110)-(1 \times 2)$ Surface
}

\author{
Angelika Kühnle • Trolle R. Linderoth • \\ Flemming Besenbacher
}

Published online: 15 October 2011

(C) Springer Science+Business Media, LLC 2011

\begin{abstract}
A pronounced enantiomeric excess of LL-cysteine dimers is observed by scanning tunneling microscopy $(\mathrm{STM})$ on the $\mathrm{Au}(110)-(1 \times 2)$ surface after partial thermal desorption/decomposition of racemic cysteine. We systematically examine several possible origins for this intriguing observation of chiral symmetry breaking, including a chiral bias of the substrate, but remain unable to identify the source.
\end{abstract}

Keywords Cysteine $\cdot \mathrm{Au}(110) \cdot$ Chirality $\cdot$ Chiral symmetry breaking $\cdot$ Scanning tunneling microscopy

\section{Introduction}

Chiral symmetry breaking in chemical, physical and biological systems has attracted considerable attention during the last decades $[1,2]$ as chiral asymmetry seems to be a prerequisite for life [3, 4]; prominent examples for the homochirality of biomolecules include the exclusive use of D-sugar molecules in the DNA backbone and L-amino acids

\footnotetext{
A. Kühnle $\cdot$ T. R. Linderoth $(\bowtie) \cdot$ F. Besenbacher Interdisciplinary Nanoscience Center (iNANO) and Department of Physics and Astronomy, Aarhus University, Ny Munkegade 118, 8000 Aarhus C, Denmark

e-mail: trolle@inano.au.dk

A. Kühnle

e-mail: kuehnle@uni-mainz.de
}

Present Address:

\section{A. Kühnle}

Institut für Physikalische Chemie, Johannes Gutenberg

Universität Mainz, Jakob-Welder-Weg 11, 55099 Mainz,

Germany as building blocks for proteins. The prebiotic origin of this imbalance is an enduring mystery, although many different sources of chiral symmetry breaking have been discussed in the literature, including the weak nuclear interaction [5] circularly polarized light [6] as well as extraterrestrial origins [7]. Spontaneous chiral symmetry breaking has been demonstrated e.g. in crystallization from solution $[8,9]$, but this is a statistical process where the opposite enantiomeric crystallites are formed with the same probability in repeated experiments, preserving overall chiral symmetry. Several mechanisms to inherit or even amplify a pre-existing enantiomeric excess have been discussed. In chemical systems this includes autocatalytic processes [10-13], and it has been speculated if such mechanisms can lead to amplification of a random, minute statistical imbalance that occurs naturally in a racemic mixture [14, 15]. Recently, phase transitions at the solution-solid interface [16] or by sublimation [17-19] have been shown to lead to amplification of small enantiomeric excesses, ultimately resulting from different solubility/volatility of racemic and enantiopure phases [19, 20]. Molecular organization on surfaces may have been involved in early formation of bio-polymers [21], and enantiospecific adsorption of amino acids onto chiral surfaces such as quartz [22] and calcite [23, 24] has been speculated to play a role in establishing the homochirality of life [25], although natural mineral surfaces are not themselves expected to exhibit a chiral bias.

The long-standing discussion concerning the fundamental origins of biomolecular homochirality has been one of several motivations for a wide variety of studies into the adsorption and organization of chiral molecules on welldefined single-crystal surfaces which have been performed during the last decade [26-32]. These studies have in particular relied on local-probe scanning tunneling 
microscopy (STM) which allows molecular and supramolecular chirality to be directly observed.

Surfaces that are otherwise achiral can be rendered globally homochiral by deposition of enantiomerically pure compounds [29]. Deposition of racemic mixtures or prochiral compounds in contrast leads to surfaces that are globally racemic [33-38]. This may involve formation of domains consisting of both enantiomers [39] but most commonly formation of two-dimensional conglomerates is observed, i.e. the molecules segregate into locally homochiral domains $[40,41]$ with domains of opposite handedness being created in equal proportion.

Several experiments concerning chiral induction, i.e. the ability to steer the chirality of molecules or assemblies towards one specific handedness, have been performed [42, 43]. Chiral symmetry on surfaces may be broken by the use of external agents in the form of a chiral solvent [44] or a magnetic field [45]. A related approach is the "sergeants and soldiers" effect where a homochiral seed induces a chiral response in target molecules as has been demonstrated in surface assemblies in a couple of instances [46-48]. Recent studies in this direction have focused on the "majority rule" where an enantiomeric excess among adsorbed molecules is amplified in the resulting supra-molecular structures $[49,50]$.

Another aspect of molecular surface chirality is the metal surfaces themselves, which can be chiral if the crystal is cut along planes of sufficiently low symmetry [51]. This may either be considered an intrinsic property of the entire surface [52], or attributed to specific chiral kink sites [53]. Chirally specific interaction between molecules and chiral metal surfaces has been demonstrated in a few instances [28, 54, 55], and the opposite effect of chiral faceting of a metallic surface through interaction with a chiral molecule has also been observed [56].

In the present paper we report an intriguing observation of chiral symmetry breaking occurring upon deposition of the amino acid cysteine on the $(1 \times 2)$ missing row reconstructed $\mathrm{Au}(110)$ surface under ultra-high vacuum conditions. When a racemic mixture of cysteine is deposited on the gold surface, a thorough statistical analysis of the recorded STM images very surprisingly reveals a pronounced enantiomeric excess of LL-cysteine dimers on the surface. Inspired by the existing literature on chiral symmetry breaking we examine and discuss a number of possible origins for this imbalance, including statistical fluctuations, chiral amplification, and an influence from chiral kink sites of the substrate surface. However, in spite of a very thorough and detailed analysis of a number of possible sources, the origin for the observed breaking of chiral symmetry for the cysteine molecules on the gold surface remains a mystery.

\section{Experimental}

The adsorption experiments were performed in an ultrahigh vacuum (UHV) chamber equipped with the homebuilt Aarhus STM [57] as well as standard facilities for sample cleaning and characterization. The $\mathrm{Au}(110)-(1 \times 2)$ crystal surfaces were cleaned by repeated cycles of $\mathrm{Ar}^{+}$ion sputtering at $1.5 \mathrm{keV}$ and annealing at $800 \mathrm{~K}$. Enantiomerically pure L- and D-cysteine as well as racemic DL-cysteine were obtained from Aldrich. The purity was stated by the supplier to be $98 \%$ (L-cysteine) greater than 99\% (D-cysteine) and greater than 98\% (DL-cysteine). The cysteine molecules were evaporated inside the vacuum chamber onto the clean gold substrate from a home-built evaporator consisting of a small glass tube wound with a metal wire for resistive heating and with a $\mathrm{Cr} / \mathrm{Al}$ thermocouple pair fused into the tube for temperature monitoring. The source-sample distance was approx. $10 \mathrm{~cm}$ and the typical evaporation time a few minutes. During evaporation, the crucible was held at specific temperatures at around $360 \mathrm{~K}$, resulting in cysteine coverages ranging from the sub monolayer regime to well beyond saturation of the first layer (the coverage in the multilayer regime is estimated to correspond to 1-3 saturated monolayers judging from the used evaporation times). The cysteine powders were used as received from the supplier, but were thoroughly outgassed in the evaporator before deposition. In all experiments, the temperature of the gold substrate during deposition was approx. $300 \mathrm{~K}$. To anneal the deposited films, the temperature of the Au crystal was brought to a specific temperature in the range of $380-420 \mathrm{~K}$ for 5-15 min by radiative heating of the back side of the sample holder. All STM images were obtained at room temperature $(\sim 300 \mathrm{~K})$ in constant current mode $\left(\mathrm{I}_{\mathrm{t}} \sim 0.1\right.$ $\left.\mathrm{nA}, \mathrm{V}_{\mathrm{t}} \sim 1.5 \mathrm{~V}\right)$ with the sample at negative bias compared to the tip.

\section{Results and Discussion}

Upon sub monolayer deposition of cysteine onto a $\mathrm{Au}(110)$ surface held at room temperature, a poorly ordered, anisotropic phase of agglomerated cysteine is obtained (not shown). Annealing this structure to $380 \mathrm{~K}$ results in the formation of characteristic double-lobe features as shown in Fig. 1a, which we reported upon previously [58] and attributed to cysteine dimers. Depending upon the chirality of the deposited enantiomerically pure cysteine, the main axis through the dimers is rotated $20^{\circ}$ clockwise (L-cysteine) or counter-clockwise (D-cysteine) with respect to the [1-10] direction, as illustrated in the STM images in Fig. 1b and c. The STM signature of the cysteine dimers thus allows them to be identified as either LL or DD. When the racemic 

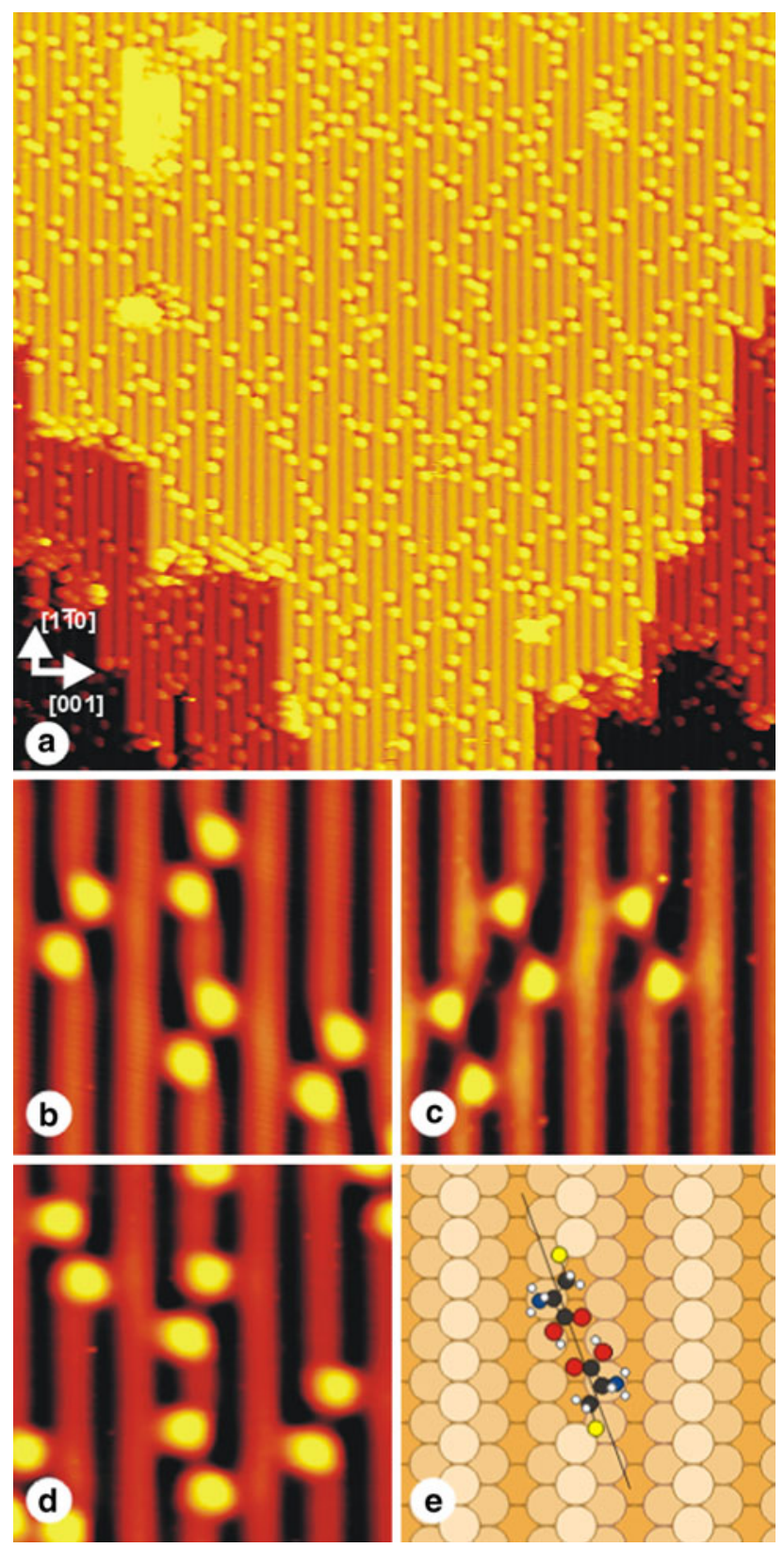

Fig. 1 a STM image showing the $\mathrm{Au}(110)-(1 \times 2)$ surface after deposition of sub monolayer coverage of racemic cysteine and annealing to $380 \mathrm{~K}$. Image size: $424 \AA \times 459 \AA$ A b Cysteine dimers observed after evaporation of L-cysteine. The dimers are exclusively rotated clockwise with respect to the [1-10] direction. Image size: $49 \AA \times 53 \AA$. c DD-cysteine dimers resembling the LL-cysteine dimers in size and appearance but exhibiting a $20^{\circ}$ counter-clockwise rotation. Image size: $49 \AA \times 53 \AA$. d Zoom into dimers formed from the racemic mixture. Both dimers rotated clockwise (LL-cysteine dimers) and counter-clockwise (DD-cysteine dimers) are observed. Image size: $49 \AA \times 53 \AA$. e Model illustrating the cysteine dimer adsorption geometry

mixture is deposited, molecular dimers are observed identical to those obtained for the enantiomerically pure compounds (Fig. 1d) while no new structures suggestive of any LD dimers were revealed. This was ascribed to exclusive formation of homochiral dimers resulting from a chiral recognition process proposed from Density Functional Theory (DFT) calculations to involve simultaneous optimisation of three interaction points for each molecule of the dimer, as illustrated in Fig. 1e. From interplay of the STM and DFT results it was concluded that the dimer resides on a four-atom vacancy in the close-packed row of the missing row reconstructed surface. The sulphur atom in cysteine is covalently bound at a bridge site next to a low-coordinated gold atom in the topmost row, the nitrogen atom coordinates via a lone pair to the gold surface, and the carboxylic group forms hydrogen bonds with the carboxylic group of the other molecule in the dimer.

In the experiments reported upon here, we followed a different protocol where racemic cysteine was initially deposited on the $\mathrm{Au}(110)-(1 \times 2)$ surface at coverages beyond saturation of the first monolayer. The sample was subsequently annealed at a slightly higher temperature of $420 \mathrm{~K}$ with the aim to desorb cysteine bound in multilayers. Figure 2a shows an STM image of the surface following this procedure. Several differences compared to the situation depicted in Fig. 1 are clearly noticeable. First, the surface is re-facetted with large rectangular terraces, in marked contrast to the characteristic "fish-scale" pattern of native $\mathrm{Au}(110)-(1 \times 2)$ where the terraces taper off in the direction of the close-packed atomic rows (compare also Fig. 4a). Second, the STM image displays a substantially higher tunneling noise, which is directly depicted as small, horizontal stripes over the close-packed Au rows. This is interpreted as species diffusing rapidly on the surface, which is confirmed upon cooling the sample to around $120 \mathrm{~K}$, where the noise vanishes and additional particles are observed (not shown). Third, several regions are observed of a new regular structure which forms elongated stripes extending more than $1000 \AA$ in the [001] direction. As can be seen in Fig. 2a, the rectangular terraces are frequently terminated by these stripes. Figure $2 \mathrm{c}$ shows a high-resolution STM image of this new phase exhibiting a $c(4 \times 2)$ superstructure. The $c(4 \times 2)$ phase is attributed to atomic sulphur adsorbed on unreconstructed $\mathrm{Au}(110)$ $(1 \times 1)$ patches as reported upon previously based on LEED measurements [59, 60]. Control experiments involving high-temperature thermal decomposition of $\mathrm{H}_{2} \mathrm{~S}$ over the $\mathrm{Au}(110)-(1 \times 2)$ surface also resulted in a structure with an identical STM signature.

Together, these observations imply that the annealing at $420 \mathrm{~K}$ results in desorption and partial decomposition of the cysteine molecules, explaining the observed $c(4 \times 2)$ structure of atomic sulphur and the rapidly diffusing molecular fragments. In addition, a considerable mass transport of $\mathrm{Au}$ atoms results in re-faceting of the surface.

From a detailed investigation of the surface terraces, we conclude that the characteristic cysteine dimer structures 


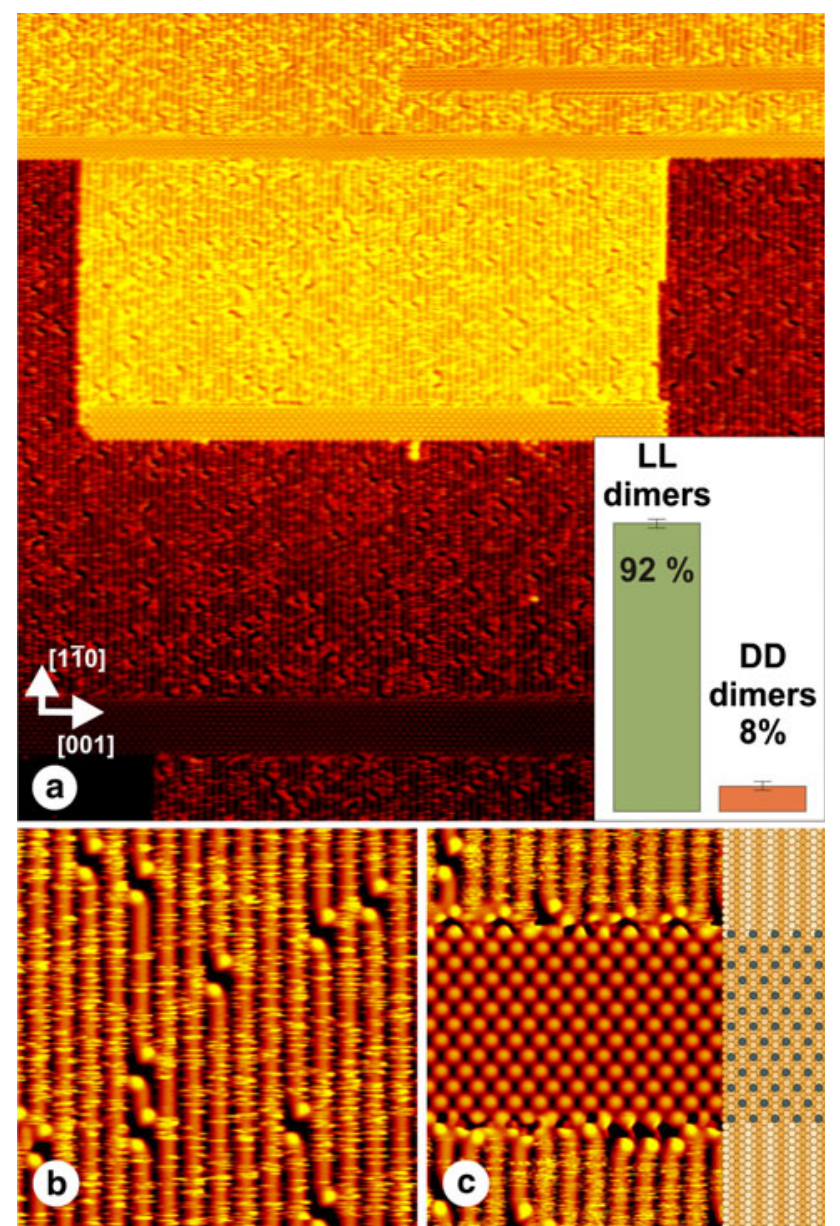

Fig. 2 a STM image showing a re-faceted terrace after highcoverage deposition of racemic cysteine and subsequent annealing of the substrate to $420 \mathrm{~K}$. Image size: $897 \AA \times 991 \AA$. Inset: Asymmetric distribution of LL and DD dimers resulting from such experiments (see text for details). b On the terrace, cysteine dimers are observed. Image size: $131 \AA \times 144 \AA$ A c Zoom into the elongated stripes observed on the surface, extending over several $1000 \AA$ in the [001] direction and showing a $\mathrm{c}(4 \times 2)$ ordering. Image size: $131 \AA \times 144 \AA$

can nevertheless still be observed, as demonstrated in Fig. 2b. These dimers are ascribed to a low number of cysteine molecules that remain intact during the annealing (the bonding model for the dimers make it unlikely that they would form from cysteine molecules that were fragmented to any significant degree, involving e.g. loss of a functional group). Inspection of Fig. $2 b$ shows that all the dimers are of the clock-wise rotated LL variant. This is very surprising since the experiment leading to the STM image in Fig. 2b involved deposition of racemic cysteine. Observations such as this led us to systematically analyze the distribution of dimers resulting from multilayer deposition of racemic cysteine and annealing to $420 \mathrm{~K}$. As shown in the inset of Fig. 2a, the highly unexpected result from analysis of 308 observed cysteine dimers is that $92 \%$ are LL dimers, whereas only $8 \%$ are DD dimers, severely departing from the $50 \%$ to $50 \%$ balance in the racemic source material.

We are thus led to the intriguing conclusion that the chiral symmetry is somehow broken in the performed experiments. In the following we present and test a number of explanations (see Fig. 3) for this highly surprising result.

One explanation might be that the excess of LL-cysteine dimers only develops locally, while there are other regions with DD dimers, maintaining a 50-50\% distribution globally on the surface. To investigate this possibility, we examined several surface areas, but an excess of LL dimers was always observed.

Secondly, the observed imbalance might result from a process randomly producing an excess of either LL- or DD-cysteine dimers globally on the surface, but with an equal probability for the two outcomes. To test this possibility we repeated the STM experiment six times, always finding an excess of LL-cysteine dimers. The probability of obtaining this result from an unbiased random process is less than $2 \%$ $\left(1 / 2^{6}\right)$ so this hypothesis can effectively be ruled out.

A third possibility is that the gold substrate is the source for the break of chiral symmetry. To affect the two
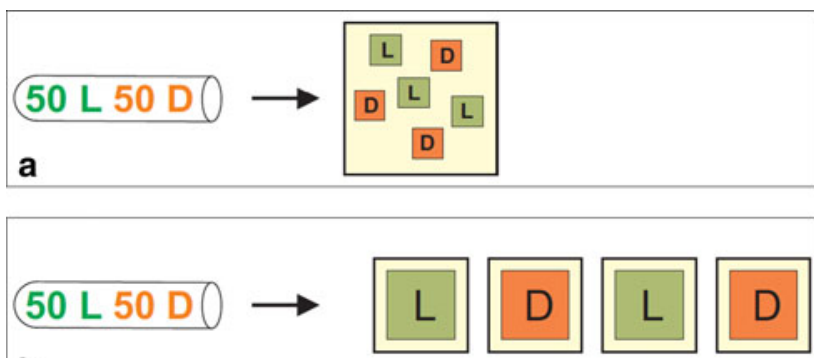

b
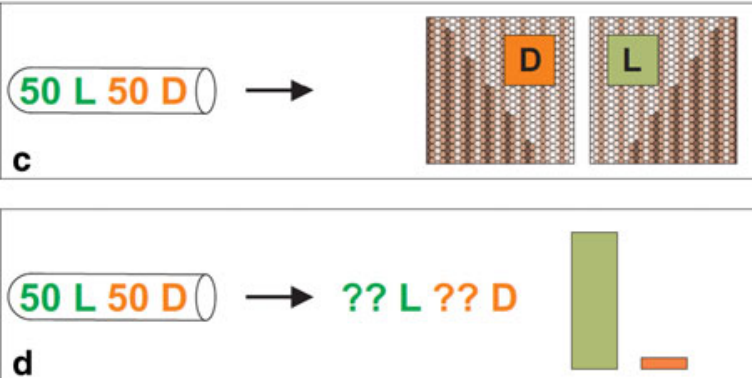

Fig. 3 Different possible scenarios that might account for the observed chiral asymmetry. a The cysteine dimers separate in L-cysteine-rich and D-cysteine-rich regions. Globally on the surface, chiral symmetry is preserved. b A random process might result in an excess of one type of enantiomer in a single experiment. However, upon repetition of the experiment, a $50-50 \%$ distribution of experiments with an excess of L- or D-cysteine restores chiral symmetry. c The chiral asymmetry might originate from chiral asymmetries of the gold substrate which may exhibit an excess of chiral kink sites if it is slightly mis-cut. d Finally, the imbalance might result from the sublimation process or the cysteine source material 
enantiomers of cysteine differently, the surface has to be chiral itself. An ideal, reconstructed (110)- $(1 \times 2)$ surface, is, however, mirror-symmetric and achiral. A step edge is not chiral either, but a kink site can constitute a chiral center [53]. An STM image of terrace terminations on the $\mathrm{Au}(110)-(1 \times 2)$ surface is shown in Fig. 4 along with illustrations of kinked step edges with mirror-image structure. The kinks are classified as either $\mathrm{S}$ or $\mathrm{R}$ depending on the order of microfacets at the kink site [53]. We previously showed in the low-coverage regime that the two enantiomers of cysteine indeed interact differently with the two types of kink sites [55]. It is thus possible that the kink sites also influence the structures in the highcoverage regime and somehow affect the thermal decomposition of cysteine. An explanation for the excess of L-cysteine dimers on the terraces might therefore be that the particular $\mathrm{Au}(110)$ surface in play exhibits an excess of kink sites of one type which could result from a slight
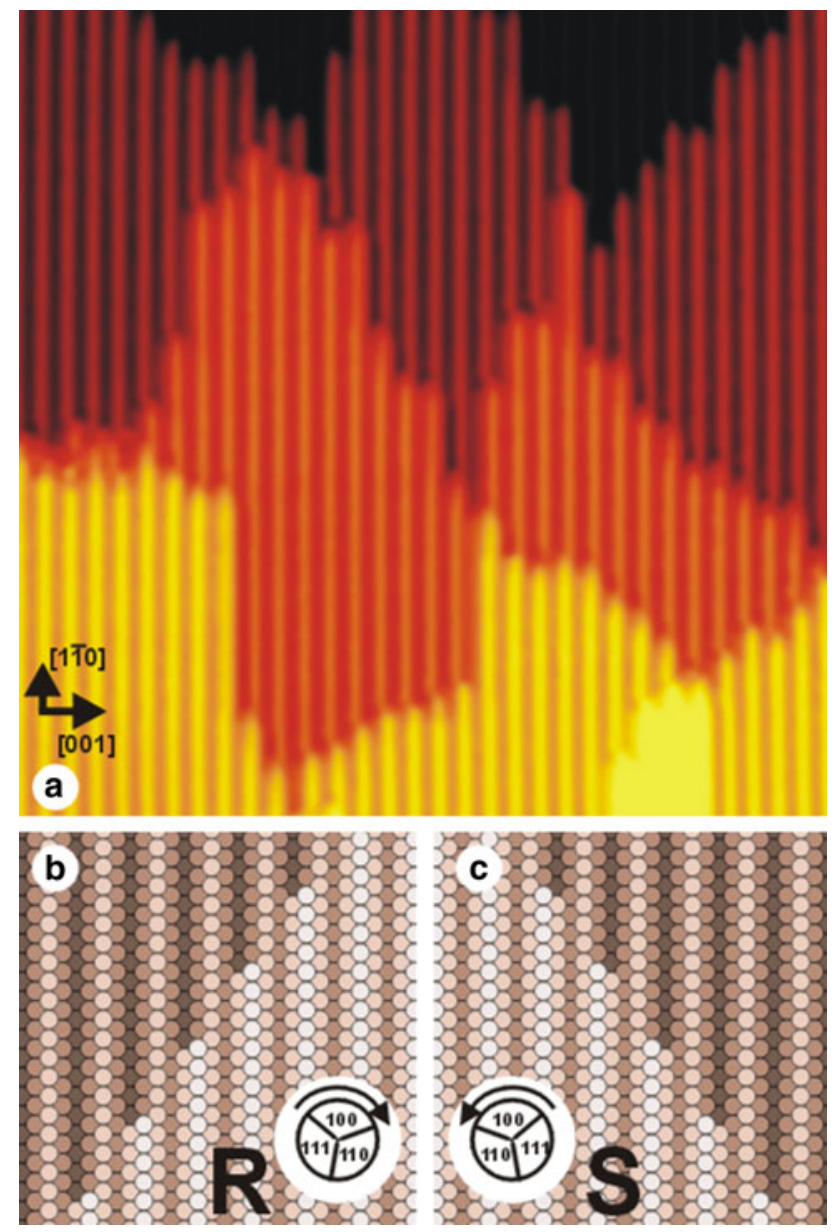

Fig. 4 a STM image of a $A u(110)-(1 \times 2)$ surface showing kink sites at terrace terminations. Image size: $277 \AA \times 299 \AA$. b, c Schematic models of kink sites which represent chiral centers at the surface. The kinks are termed $\mathrm{R}$ or $\mathrm{S}$ depending on the order of microfacets at the kink site [53] miscut of the crystal or perhaps even from chirally biased re-facetting induced during initial experiments with enantiomerically pure cysteine [56].

To investigate this idea, two chiral and mirror image gold surfaces were prepared by deliberate mis-orientation before polishing: We started with two gold crystals cut to expose the (110) surface plane. Both crystals were first rotated by $0.5^{\circ}$ about the [001] axis (to create step edges). Secondly, the two crystals were rotated by $\pm 0.5^{\circ}$, respectively, around the [1-10] axis to produce chiral, kinked surfaces that are mirror images of each other. A third crystal polished with the best possible alignment along the (110) plane was prepared for control. Mis-orientation by $0.5^{\circ}$ is at the limit of the precision for aligning the gold crystals, but was chosen since it for an idealised truncation of the bulk structure should yield kink site terminated terraces with a width of $\sim 110 \AA$. Smaller terrace sizes were judged inconvenient for identifying the cysteine dimers on the terraces. The two slightly misaligned $\mathrm{Au}(110)$ surfaces were examined by recording STM images of random areas within the $2 \times 2 \mu \mathrm{m}^{2}$ range accessible by our STM scanner head, and also for several macroscopially different positions on the sample. The resulting images (typically $100 \times 100 \mathrm{~nm}^{2}$ in size) were analyzed with respect to the chirality of the observed kink sites and were classified as $\mathrm{S}$ or $\mathrm{R}$ kinked depending on the dominating type of kink site. On one of the two misaligned surfaces, 101 images from nine macroscopically different areas were evaluated, revealing 76 areas predominantly exhibiting $\mathrm{S}$ kinks and 25 areas with $\mathrm{R}$ kinks being predominant, corresponding to $75 \%$ and $25 \%$, respectively. The other surface with opposite misalignment was examined at 126 different places and showed 29 areas (23\%) with $\mathrm{S}$ and 97 areas (77\%) with R kinks. The special polishing of the samples thus indeed seems to yield the desired chiral asymmetry of the surfaces.

The experiment was then repeated by depositing racemic cysteine on these three surfaces in the multilayer regime and the distribution of cysteine dimers observed after annealing at $420 \mathrm{~K}$ was analysed. The results are reported in Table 1, showing LL dimer fractions in the range from $70 \%$ to $87 \%$. A clear excess of LL-cysteine

Table 1 Distribution of DD- and LL-cysteine dimers on four different gold surfaces (see text) after high-coverage deposition and subsequent annealing at $420 \mathrm{~K}$

\begin{tabular}{lcrr}
\hline Surface & DD dimers & \multicolumn{1}{c}{ LL dimers } & Sum \\
\hline Au(110) original & $26(8 \%)$ & $282(92 \%)$ & 308 \\
$\mathrm{Au}(110) \mathrm{S}$ & $173(13 \%)$ & $1163(87 \%)$ & 1336 \\
$\mathrm{Au}(110) \mathrm{R}$ & $49(27 \%)$ & $130(73 \%)$ & 179 \\
$\mathrm{Au}(110)$ reference & $147(30 \%)$ & $351(70 \%)$ & 498 \\
\hline
\end{tabular}


dimers is thus observed on all three surfaces, irrespective of the chiral nature of the dominating kink sites. A chiral bias of the Au(110) substrate can thus be ruled out as the source of the chiral symmetry break.

A final candidate to consider is the deposition process and the racemic cysteine source material itself. A standard measurement of the optical rotation of a solution of the used LD-cysteine powder confirmed it to be an exact $50-50 \%$ mixture to a precision of a few tenths of a per cent and thus gave no indication of chiral bias in the source material. In a series of experiments we returned to the original preparation protocol with sub-monolayer deposition of the racemic mixture and annealing to $380 \mathrm{~K}$ to form the dimers (compare Fig. 1). Table 2 summarizes the results from an extensive statistical analysis of the distribution of cysteine dimers resulting from this procedure on all four surfaces examined. Interestingly, we found also for this protocol a slight excess of L-cysteine, relatively independent of the surface used, with the fraction of LL-cysteine dimers being $56 \%$ on average. This slight excess was not recognised in our earlier experiments [58] as an extensive data material is required to detect it with statistical significance. One possible interpretation of this result is that the unknown mechanism leading to an L-cysteine excess is at play already in this regime of lower coverage and lower annealing temperature, but to a lesser extent than in the experiments with annealing to $420 \mathrm{~K}$. Another possibility is that the evaporation source initially delivers this slight enantiomeric excess of L-cysteine to the surface and that it is subsequently amplified in the high-coverage experiments during the annealing at $420 \mathrm{~K}$ where there is pronounced desorption/decomposition.

One might speculate that an enantiomeric imbalance in the deposition could result from having an unequal number of crystallites of pure L and D cysteine in the relatively small sample volume loaded into the evaporator, or from an excess of L-cysteine crystallites close to the surface of the evaporation source, leading to a bias in the evaporated molecules. However, this explanation can be ruled out since cysteine belongs to the class of racemic compounds where the racemate crystallises with both enantiomers in the unit cell [61], and pure DL-cysteine, as used in the present experiments, is therefore expected to have an exact

Table 2 Distribution of DD- and LL-cysteine dimers on four different gold surfaces (see text) after submonolayer deposition and subsequent annealing at $380 \mathrm{~K}$

\begin{tabular}{lrrr}
\hline Surface & \multicolumn{1}{c}{ DD dimers } & \multicolumn{1}{c}{ LL dimers } & Sum \\
\hline $\mathrm{Au}(110)$ original & $651(42 \%)$ & $892(58 \%)$ & 1543 \\
$\mathrm{Au}(110) \mathrm{S}$ & $1194(44 \%)$ & $1546(56 \%)$ & 2740 \\
$\mathrm{Au}(110) \mathrm{R}$ & $268(46 \%)$ & $320(54 \%)$ & 588 \\
$\mathrm{Au}(110)$ reference & $644(46 \%)$ & $765(54 \%)$ & 1409 \\
\hline
\end{tabular}

$50-50 \%$ distribution of the two enantiomers at the molecular level.

With the aim to perform a deposition that was deliberately chirally biased, we prepared a mechanical mixture of $70 \%$ D- and $30 \%$ L-cysteine from the enantiomerically pure powders and loaded this in a single evaporation source. Deposition was performed using our standard procedure and the experiment was carried out in the low-coverage regime with subsequent annealing at $380 \mathrm{~K}$. The expectation in this case was to observe the initial 70D:30L enantiomeric ratio also after deposition, or possibly a distribution enhanced in $\mathrm{D}$, demonstrating an amplification mechanism towards the major enantiomer. Instead, a detailed analysis of 649 cysteine dimers observed in this experiment somewhat surprisingly revealed an essentially equal ratio of the two enatiomers with $312(48 \%)$ DD and 337 (52\%) LL dimers. However, recent investigations [19] in fact suggest that no enantiomeric excess in the sublimate is exactly the anticipated result from sublimation of such a "kinetic conglomerate" [20]; the deposited amount is determined solely by the vapour pressures of the enantiomerically pure crystallites, which are identical, while the amount of material of the two phases in the source material in the idealized case is of no importance. ${ }^{1}$ We therefore cannot conclude from this experiment whether there is a chiral amplification mechanism on the surface following deposition.

Chiral amplification on the surface during the postdeposition annealing might result from an autocatalytic process $[11,12]$ or through a majority rule effect $[49,50]$. In this context it is also particularly relevant to note recent results demonstrating that a small enantiomeric excess in amino acid samples can be amplified by sublimation, leading to a substantially higher enantiomeric excess in the sublimate [17-20]. The present experiments involve two subsequent sublimations, one from the crucible during deposition and one from the surface in the experiments with multilayer deposition and annealing at $420 \mathrm{~K}$, although it should be noted that the situation in the latter case is complicated by competition with thermal decomposition and it is not the sublimate but rather the material left on the surface which is examined. Furthermore, even if there is a relevant mechanism for chiral amplification of a small enantiomeric excess, it does not explain where in the present experiments the initial break of chiral symmetry might originate from.

\footnotetext{
$\overline{1}$ A chiral bias in the deposited material could be achieved by either depositing different amounts of enantiomerically pure cysteine from two separate sources, or possibly by using a single source loaded with racemic DL cysteine enriched with enantiomerically pure $\mathrm{L}$ or $\mathrm{D}$ cysteine in which case the enantiomeric ratio in the deposit is determined by the vapour pressures of the racemate and the enantiopure crystals which in general are expected to be different [19].
} 
Previous cases of apparent chiral symmetry breaking in autocatalytic, repetitive reactions have been attributed to unidentified trace impurities $[14,15]$. We tentatively suggest that unknown impurities in the racemic cysteine source material may also be responsible for the apparent case of symmetry breaking observed here.

\section{Conclusion}

In summary, we have investigated the adsorption of the naturally occurring chiral amino acid cysteine on the missing row reconstructed $\mathrm{Au}(110)-(\times 2)$ surface using scanning tunneling microscopy. Following deposition of racemic cysteine we very surprisingly observe a pronounced enantiomeric excess of LL-cysteine dimers on the surface, apparently breaking the chiral symmetry. We have examined and discussed a number of possible origins for this intriguing observation of chiral symmetry breaking, including statistical effects, a chiral bias of the Au surface and possible mechanisms for chiral bias or amplification during deposition or post-deposition annealing. However the mechanism or source for the break of chiral symmetry remains unidentified. We hope that our extremely surprising finding and the efforts undertaken to elucidate it may stimulate further work within the fascinating field of chiral symmetry breaking.

Acknowledgments We acknowledge financial support from the Danish Council for Independent Research I Natural Sciences, The Villum Kahn Foundation, The Carlsberg Foundation, the European Research Council (ERC) and the Danish National Research Foundation for support to the Sino-Danish Center for Molecular Nanostructures on Surfaces. AK acknowledges financial support from the German Research Foundation (DFG) through the Emmy Noetherprogram KU 1980/1-3.

\section{References}

1. Avalos M, Babiano R, Cintas P, Jimenez JL, Palacios JC (2010) Tetrahedron Asymmetr 21:1030

2. Ball P (2007) Chem World 4:30

3. Bonner WA (1995) Origins Life Evol Biosph 25:175

4. Bonner WA (1996) AIP Conf Proc 379:17

5. Kovacs KL (1979) Origins Life 9:219

6. Goodman G, Gershwin ME (2006) Exp Biol Med (Maywood, NJ) 231:1587

7. Strasdeit H (2005) ChemBioChem 6:801

8. Kondepudi D, Kaufman R, Singh N (1990) Science 250:975

9. Buhse T, Durand D, Kondepudi D, Laudadio J, Spilker S (2000) Phys Rev Lett 84:4405

10. Frank FC (1953) Biochim Biophys Acta 11:459

11. Soai K, Shibata T, Morioka H, Choji K (1995) Nature (London) 378:767

12. Blackmond DG, McMillan R, Ramdeehul S, Schorm A, Brown JM (2001) J Am Chem Soc 123:10103
13. Saghatelian A, Yokobayashi Y, Soltani K, Ghadiri R (2001) Nature 409:797

14. Singleton DA, Vo LK (2002) J Am Chem Soc 124:10010

15. Siegel JS (2002) Nature (London) 419:346

16. Klussmann M, Iwamura H, Mathew SP, Wells DH, Pandya U, Armstrong A, Blackmond DG (2006) Nature 441:621

17. Perry RH, Wu CP, Nefliu M, Cooks RG (2007) Chem Commun 1071

18. Fletcher SP, Jagt RBC, Feringa BL (2007) Chem Commun 2578

19. Bellec A, Guillemin JC (2010) Chem Commun 46:1482

20. Blackmond DG, Klussmann M (2007) Chem Commun 3990

21. Sowerby SJ, Heckl WM (1998) Origins Life Evol Biosph 28:283

22. Soai K, Osanai S, Kadowaki K, Yonekubo S, Shibata T, Sato I (1999) J Am Chem Soc 121:11235

23. Hazen RM, Filley TR, Goodfriend GA (2001) Proc Natl Acad Sci 98:5487

24. Hazen RM (2001) Sci Am 284:76

25. Hazen RM, Sholl DS (2003) Nat Mater 2:367

26. Raval R (2009) Chem Soc Rev 38:707

27. Elemans J, De Cat I, Xu H, De Feyter S (2009) Chem Soc Rev $38: 722$

28. Gellman AJ (2010) ACS Nano 4:5

29. Lorenzo MO, Baddeley CJ, Muryn C, Raval R (2000) Nature 404:376

30. Raval R (2002) J Phys: Condensed Matter 14:4119

31. Chen Q, Richardson NV (2004) Annu Rep Prog Chem C 100:313

32. Ernst K-H (2006) Top Curr Chem 265:209

33. Bohringer M, Morgenstern K, Schneider WD, Berndt R, Mauri F, De Vita A, Car R (1999) Phys Rev Lett 83:324

34. Weckesser J, De Vita A, Barth JV, Cai C, Kern K (2001) Phys Rev Lett 87:096

35. Chen Q, Richardson NV (2003) Nat Mater 2:324

36. Weigelt S, Busse C, Petersen L, Rauls E, Hammer B, Gothelf KV, Besenbacher F, Linderoth TR (2006) Nat Mater 5:112

37. Dmitriev A, Spillmann H, Stepanow S, Strunskus T, Woell C, Seitsonen AP, Lingenfelder M, Lin N, Barth JV, Kern K (2006) ChemPhysChem 7:2197

38. Stevens F, Dyer DJ, Walba DM (1996) Angew Chem Int Ed Engl 35:900

39. Fasel R, Parschau M, Ernst K-H (2006) Nature (London) 439:449

40. Eckhardt CJ, Peachey NM, Swanson DR, Takacs JM, Khan MA, Gong X, Kim J-H, Wang J, Uphaus A (1993) Nature 362:614

41. Eralp T, Shavorskiy A, Zheleva ZV, Held G, Kalashnyk N, Ning YX, Linderoth TR (2010) Langmuir 26:18841

42. Ernst K-H (2008) Curr Opin Colloid Interface Sci 13:54

43. Palmans ARA, Meijer EW (2007) Angew Chem Int Ed 46:8948

44. Katsonis N, Xu H, Haak RM, Kudernac T, Tomovic Z, George S, Van der Auweraer M, Schenning A, Meijer EW, Feringa BL, De Feyter S (2008) Angew Chem Int Ed 47:4997

45. Berg AM, Patrick DL (2005) Angew Chem Int Ed 44:1821

46. Parschau M, Romer S, Ernst K-H (2004) J Am Chem Soc 126:15368

47. Parschau M, Kampen T, Ernst KH (2005) Chem Phys Lett 407:433

48. Roth C, Passerone D, Ernst KH (2010) Chem Commun 46:8645

49. Fasel R, Parschau M, Ernst K-H (2006) Nature 439:449

50. Haq S, Liu N, Humblot V, Jansen APJ, Raval R (2009) Nat Chem 1:409

51. McFadden CF, Cremer PS, Gellman AJ (1996) Langmuir 12:2483

52. Jenkins SJ, Pratt SJ (2007) Surf Sci Rep 62:373

53. Ahmadi A, Attard G, Feliu J, Rodes A (1999) Langmuir 15:2420

54. Horvath JD, Gellman AJ (2002) J Am Chem Soc 124:2384

55. Kühnle A, Linderoth TR, Besenbacher F (2006) J Am Chem Soc 128:1076

56. Zhao XY (2000) J Am Chem Soc 122:12584 
57. Besenbacher F, Lægsgaard E, Mortensen K, Nielsen U, Stensgaard I (1988) Rev Sci Instrum 59:1035

58. Kühnle A, Linderoth TR, Hammer B, Besenbacher F (2002) Nature 415:891
59. Jaffey DM, Madix RJ (1991) Surf Sci 258:359

60. Kostelitz M, Domagne JL, Oudar J (1973) Surf Sci 34:431

61. Luger P, Weber M (1999) Acta Crystallogr C 55:1882 